\title{
System identification of a building from multiple seismic records
}

\author{
Hasan S. Ulusoy ${ }^{1, *, \dagger}$, Maria Q. Feng ${ }^{1}$ and Paul J. Fanning ${ }^{2}$ \\ ${ }^{1}$ Department of Civil and Environmental Engineering, University of California, Irvine, CA 92697, U.S.A. \\ ${ }^{2}$ School of Architecture, Landscape and Civil Engineering, University College Dublin, Belfield, Dublin 4, Ireland
}

\begin{abstract}
SUMMARY
This paper describes the identification of finite dimensional, linear, time-invariant models of a 4-story building in the state space representation using multiple data sets of earthquake response. The building, instrumented with 31 accelerometers, is located on the University of California, Irvine campus. Multiple data sets, recorded during the 2005 Yucaipa, 2005 San Clemente, 2008 Chino Hills and 2009 Inglewood earthquakes, are used for identification and validation. Considering the response of the building as the output and the ground motion as the input, the state space models that represent the underlying dynamics of the building in the discrete-time domain corresponding to each data set are identified. The time-domain Eigensystem Realization Algorithm with the Observer/Kalman filter identification procedure are adopted in this paper, and the modal parameters of the identified models are consistently determined by constructing stabilization diagrams. The four state space models identified demonstrate that the response of the building is amplitude dependent with the response frequency and damping, being dependent on the magnitude of ground excitation. The practical application of this finding is that the consistency of this building response to future earthquakes can be quickly assessed, within the range of ground excitations considered $(0.005 g-0.074 g)$, for consistency with prior response- this assessment of consistent response is discussed and demonstrated with reference to the four earthquake events considered in this study. Inclusion of data sets relating to future earthquakes will enable the findings to be extended to a wider range of ground excitation magnitudes. Copyright (C) 2010 John Wiley \& Sons, Ltd.
\end{abstract}

Received 22 September 2009; Revised 25 June 2010; Accepted 5 July 2010

KEY WORDS: system identification; earthquake response; building structures; modal parameters

\section{INTRODUCTION}

System identification deals with building mathematical models from observations. This simple yet general statement makes system identification a multidisciplinary research area since mathematical models are approximate representations of physical phenomena or processes, and essential for simulation, prediction and design in engineering and science. The theory, techniques and applications in the area have been developed over several years mostly by automatic control and system theory disciplines. The techniques that depend on the properties of the models to be estimated can be classified in many ways: linear, nonlinear, hybrid, parametric, nonparametric, etc. For instance, in parametric identification techniques, a certain model structure is first adopted. The choice of the model structure is often influenced by the intended use of the model and the simplicity of the estimation algorithm. The model is then characterized by a set of unknown parameters. The model provides physical insights if these adjustable parameters are chosen to be physical properties of

\footnotetext{
${ }^{*}$ Correspondence to: Hasan S. Ulusoy, Department of Civil and Environmental Engineering, University of California, Irvine, CA 92697, U.S.A.

†E-mail: hulusoy@uci.edu
} 
the system. In what follows, the identification problem reduces to the estimation of unknown parameters guided by observation data [1].

Identification techniques require excitation (input) and response (output) measurements for a complete determination of a model. In order to obtain input and output data, one needs to perform an experiment/test on the system/structure under study. For instance, in modal testing, it is a common practice to excite the test structure by applying measurable excitations at several points, then collect response data at the sensor locations [2]. However, many civil engineering structures are difficult to excite artificially due to their large size, geometry and location. Equally a large amount of external energy is needed to excite an entire structure at a desired level of vibration. Besides, even if artificial excitation is provided, civil engineering structures, which by necessity are tested in situ, continue to be excited by other unmeasurable forces (wind, waves and traffic for example) and it is thus not possible to confidently declare that any measured response is necessarily only due to any artificial excitation provided. In order to deal in part with such difficulties, some identification algorithms have been developed based on output only vibration data due to ambient and environmental forces, i.e. traffic, wind, etc. [3-5]. In this case, the measurement of the excitation is circumvented, and a modal model consisting of a set of modal parameters can be identified. Such modal parameters (natural frequencies, damping ratios, mode shapes) are classified as operational parameters as they are not normalized relative to a known excitation. A literature review on operational modal analysis is given in [6]. For civil engineering structures, strong ground motions can be considered as excitation for system identification purpose. These events can provide input data as well as output data if structures are adequately instrumented. In this case, external energy is provided naturally, and the excitation is regarded to be the inertial force that can be measured indirectly by the sensors at the ground.

Instrumentation of civil engineering structures, such as highway and suspension bridges, office buildings and TV towers, has motivated researchers to develop and validate identification techniques and algorithms for structural identification, health monitoring and damage detection purposes. The practical and realistic way to validate such techniques is to use the response data of the fullscale structures to actual events. Multiple data sets, if available, from different events at various excitation levels are particularly valuable to evaluate the identified models and compare their parameters. The frequencies of a 9-story building, the Millikan Library, obtained from ambient and forces vibration tests, and earthquake response data were compared in [7]. The amplitude and time-dependent changes of the apparent frequency of a 7-story building, the Van Nuys Hotel, were addressed using multiple earthquake response data sets and two ambient tests $[8,9]$. The fundamental fixed-base frequency changes of the Van Nuys Hotel building were discussed by analyzing the records of 11 earthquakes [10]. The effect of different input configurations on the modal parameters was discussed [11]. Using the earthquake response of real structures, several other applications of identification techniques to civil engineering structures have been reported [12-15].

A time-domain method, called the Eigensystem Realization Algorithm (ERA), has been developed for modal parameter identification using the impulse response of a system [16]. For the case when the impulse response is not measured directly, the method was later extended by adding a procedure, called Observer/Kalman Filter IDentification (OKID), to recover the impulse response from input-output data [17]. The ERA/OKID originates from the Ho-Kalman realization algorithm [18]. The method presents a unified procedure to build state space models for linear time-invariant systems from the observation data. Unlike the modal identification algorithms in which the modal parameters merely are of interest, the method provides a model that can be used for a wide range of subsequent different types of structural analysis. The applications of the method to civil engineering structures were reported in $[19,20]$.

The objective of this study is to investigate the inherent differences and similarities in dynamic response of the superstructure from event to event. In this study, identification of the state space models for a 4-story building is extended for multiple seismic records. A model that represents the underlying dynamics of the building is first identified from a single data set, the estimation data set. The identified model is then tested through the other data sets, the validation data sets. 
The determination of the model order is discussed. The modal parameters are extracted from the identified models by constructing stabilization diagrams. The excitation-dependent variation of the modal parameters is also discussed. Instrumentation of the building and details of the recorded ground motions are described.

\section{METHODOLOGY}

\subsection{Mathematical descriptions of a linear system}

Civil engineering structures are distributed parameter systems of infinite dimension. Discretization in space leads to finite dimensional systems, and provides convenient and practical approaches to the analysis and synthesis of complex systems. After discretization in time and space, a finite dimensional, linear, time-invariant structural system can be described by

$$
\begin{aligned}
\mathbf{x}(k+1) & =\mathbf{A} \mathbf{x}(k)+\mathbf{B u}(k) \\
\mathbf{y}(k) & =\mathbf{C} \mathbf{x}(k)+\mathbf{D u}(k)
\end{aligned}
$$

where $\mathbf{x} \in \mathbb{R}^{n \times 1}$ is the state vector, $\mathbf{y} \in \mathbb{R}^{m \times 1}$ is the measurement vector, $\mathbf{u} \in \mathbb{R}^{r \times 1}$ is the excitation vector, whereas $\mathbf{A} \in \mathbb{R}^{n \times n}$ is the state transition matrix, $\mathbf{B} \in \mathbb{R}^{n \times r}$ is the input matrix, $\mathbf{C} \in \mathbb{R}^{m \times n}$ is the output matrix, $\mathbf{D} \in \mathbb{R}^{m \times r}$ is the feedforward matrix and the variable $k$ indicates time dependency. The transfer function of the system described by Equations (1) is

$$
\mathbf{H}(z)=\mathbf{D}+\mathbf{C}(z \mathbf{I}-\mathbf{A})^{-1} \mathbf{B}=\mathbf{D}+\sum_{k=1}^{\infty} z^{-k} \mathbf{C A}^{k-1} \mathbf{B}, \quad z=\mathrm{e}^{\mathrm{j} \omega \Delta t} \in \mathbb{C}, \quad \mathrm{j}=\sqrt{-1}
$$

where $\omega$ is the frequency variable and $\Delta t$ is the sampling period. A set of differential/difference equations, for instance, Equations (1), that describes the internal as well as the terminal behavior of a system may be called the internal or the state-variable description of a system. On the other hand, the transfer function that describes only the terminal property of a system may be called the external or the input-output description of a system. In this study, given the external description of the system, i.e., a finite samples of the input and output sequences $\{\mathbf{u}(k)\}$ and $\{\mathbf{y}(k)\}$, $k=0, \ldots, l-1$, the objective is to find a state-variable description of the system with the smallest dimension. More explicitly, the aim is to find the system matrices $\mathbf{A}, \mathbf{B}, \mathbf{C}, \mathbf{D}$ in Equations (1) given the sequences $\{\mathbf{u}(k)\}$ and $\{\mathbf{y}(k)\}, k=0, \ldots, l-1$. Analogous to the fundamental solution in mathematics, the expressions $\mathbf{C A}^{k-1} \mathbf{B}, k=1, \ldots, \infty$, in the transfer function are called the impulse response in engineering, also known as the Markov parameters [18].

\subsection{Identification of observer and system Markov parameters}

The direct identification of the Markov parameters of the system described by Equations (1) is not immediate if the damping in the system is low and the data length is not long enough. The slow impulse response decay requires relatively a large number of the Markov parameters to be identified. However, from the mathematical theory of the realization problem, it is also known that all the system Markov parameters are not independent if data have a finite dimensional realization [18]. Let $\mathbf{Y}_{k}$ denote the system Markov parameters, $\mathbf{C A}^{k-1} \mathbf{B}$, and then the Cayley-Hamilton theorem implies that the Markov parameters can be written in a recursive form as

$$
\mathbf{Y}_{n+k+1}+\sum_{i=1}^{n} \alpha_{n-i+1} \mathbf{Y}_{k+i}=\mathbf{0}, \quad \alpha_{i} \in \mathbb{R}, \quad k=0, \quad 1, \ldots, \infty
$$

if the data have a finite dimensional, say $n$, realization. Hence, it is desirable to determine only a certain number of the Markov parameters. The above-mentioned considerations lead one to first 
identify the Markov parameters of an observer system, then identify the system Markov parameters afterwards. Let the observer system associated with Equations (1) be described by

$$
\begin{aligned}
\hat{\mathbf{x}}(k+1) & =\mathbf{A} \hat{\mathbf{x}}(k)+\mathbf{B u}(k)+\mathbf{M} \varepsilon(k) \\
\mathbf{y}(k) & =\mathbf{C} \hat{\mathbf{x}}(k)+\mathbf{D u}(k)+\varepsilon(k)
\end{aligned}
$$

where $\hat{\mathbf{x}}(k)$ is the state estimation, $\varepsilon(k)$ is the residual error and $\mathbf{M}$ is the observer gain that can be arbitrarily assigned such that the impulse response decay of the observer system is fast. In such a system, the input-output relation can be described by a limited number of the Markov parameters, say $p$, if $\mathbf{M}$ is chosen appropriately [17]. The observer system, Equations (4), can also be described by

$$
\mathbf{y}(i)=\mathbf{C}(\mathbf{A}-\mathbf{M C})^{p} \hat{\mathbf{x}}(i-p)+\sum_{k=1}^{p} \hat{\mathbf{Y}}_{k}^{(2)} \mathbf{y}(i-k)+\sum_{k=1}^{p} \hat{\mathbf{Y}}_{k}^{(1)} \mathbf{u}(i-k)+\mathbf{D u}(i)+\varepsilon(i)
$$

where $\hat{\mathbf{Y}}_{k}^{(1)}=\mathbf{C}(\mathbf{A}-\mathbf{M C})^{k-1}(\mathbf{B}-\mathbf{M D}), \hat{\mathbf{Y}}_{k}^{(2)}=\mathbf{C}(\mathbf{A}-\mathbf{M C})^{k-1} \mathbf{M}$ are defined as the block partitions of the observer Markov parameters. The key idea is to suppress the first term on the right-hand side of Equation (5) by assigning the gain matrix $\mathbf{M}$ such that $(\mathbf{A}-\mathbf{M C})^{k-1} \approx \mathbf{0}$ for $k>p$, where $p$ is a sufficiently large number. Such a gain matrix would justify the truncation of this term in Equation (5), and result in $\hat{\mathbf{Y}}_{k}^{(1)} \approx \mathbf{0}$ and $\mathbf{Y}_{k}^{(2)} \approx \mathbf{0}$ for $k>p$. Then, Equation (5) becomes AutoRegressive with eXogenous input (ARX) model and its coefficients can be estimated by the Least-Squares method [1]. Let $\mathbf{Y}_{0}$ be defined by $\mathbf{D}$. Once the observer Markov parameters are estimated, the system Markov parameters, including $p$ independent ones only, can be then extracted from the recursive relation

$$
\mathbf{Y}_{k}=\hat{\mathbf{Y}}_{k}^{(1)}+\sum_{i=1}^{k} \hat{\mathbf{Y}}_{i}^{(2)} \mathbf{Y}_{k-i} \quad \text { for } k=1, \ldots, \infty
$$

\subsection{Eigensystem Realization Algorithm}

Once the system Markov parameters, $\mathbf{Y}_{k}$, are determined as explained in Section 2.2, the system matrices A, B and $\mathbf{C}$ can be determined by the ERA [16]. The algorithm begins by forming a block Hankel matrix that consists of the system Markov parameters

$$
\mathscr{H}(k-1)=\left[\begin{array}{cccc}
\mathbf{C A}^{k-1} \mathbf{B} & \mathbf{C A}^{k} \mathbf{B} & \ldots & \mathbf{C A}^{k+\beta-2} \mathbf{B} \\
\mathbf{C A}^{k} \mathbf{B} & \mathbf{C A}^{k+1} \mathbf{B} & \ldots & \mathbf{C A}^{k+\beta-1} \mathbf{B} \\
\cdots & \ldots & \ldots & \ldots \\
\mathbf{C A}^{k+\alpha-2} \mathbf{B} & \mathbf{C A}^{k+\alpha-1} \mathbf{B} & \ldots & \mathbf{C A}^{k+\alpha+\beta-3} \mathbf{B}
\end{array}\right]
$$

where $\alpha$ and $\beta$ are two parameters that control the row and column dimension of the block Hankel matrix, $\mathscr{H}$, respectively. The system order is not known a priori. The singular value decomposition of $\mathscr{H}(0)$ is used to determine the model order and system matrices afterwards. In theory, the number of nonzero singular values is the model order. In practice, however, all the singular values appear to be nonzero due to noise and other effects. In this case, one seeks an obvious gap between two successive singular values. If such a gap exists, the number of the significant singular values, say $n$, is chosen to be the model order, and $\mathscr{H}(0)$ is then approximated by retaining the significant singular values and truncating the rest as follows:

$$
\mathscr{H}(0)=\mathscr{O} \mathscr{C}=\mathbf{U} \boldsymbol{\Sigma} \mathbf{V}^{\mathrm{T}} \approx\left[\begin{array}{ll}
\mathbf{U}_{1} & \mathbf{U}_{2}
\end{array}\right]\left[\begin{array}{cc}
\boldsymbol{\Sigma}_{n} & \mathbf{0} \\
\mathbf{0} & \mathbf{0}
\end{array}\right]\left[\begin{array}{c}
\mathbf{V}_{1}^{\mathrm{T}} \\
\mathbf{V}_{2}^{\mathrm{T}}
\end{array}\right]=\mathbf{U}_{1} \boldsymbol{\Sigma}_{n} \mathbf{V}_{1}^{\mathrm{T}}
$$

where $\mathcal{O}$ and $\mathscr{C}$ are the extended observability and controllability matrices, and can be determined by Equations (9) and (10), respectively

$$
\boldsymbol{O}=\mathbf{U}_{1} \boldsymbol{\Sigma}_{n}^{1 / 2}
$$




$$
\mathscr{C}=\Sigma_{n}^{1 / 2} \mathbf{V}_{1}^{\mathrm{T}}
$$

Finally, the system matrices are given by

$$
\begin{aligned}
& \mathbf{A}=\boldsymbol{\Sigma}_{n}^{-1 / 2} \mathbf{U}_{1}^{\mathrm{T}} \mathscr{H}(1) \mathbf{V}_{1} \boldsymbol{\Sigma}_{n}^{-1 / 2} \\
& \mathbf{B}=\mathscr{C}(1: n, 1: r) \\
& \mathbf{C}=\mathscr{O}(1: m, 1: n)
\end{aligned}
$$

Further details of the procedure described in Sections 2.2 and 2.3 can be found in $[16,17,21]$.

\subsection{Identification of the modal parameters}

A set of modal parameters can be determined after a state space model is identified from inputoutput data. Given a model, the determination of the modal parameters is a straightforward eigenvalue problem. The key issue here is the choice of the model order, which is not known a priori. The choice of model order determines the number of modal parameters, and often has effect on their results. In theory, as mentioned in Section 2.3, the model order can be determined by looking at the singular value spectrum of the Hankel matrix. The number of the significant singular values is the model order. In practice, however, the determination of model order requires further analysis because of noise and discretization errors. For real data sets, the choice of the number of the significant singular values requires prior knowledge of the system or personal judgment because there is often no clear gap to separate some singular values from the rest. The determination of the model order then becomes subjective by looking only at singular values plot of the Hankel matrix $[19,20]$. The choice of model order can be further refined by the construction of the stabilization diagram $[2,5]$. Such a diagram simply helps tracking the behavior of the modal parameters versus the model order, and it is a useful tool to identify actual modes, reject spurious or noisy modes based on certain criteria. The implementation of the stabilization diagram starts with identifying a model with a relatively high order. The model order is then reduced sequentially and the modal parameters (the frequencies, the damping ratios and the mode shapes) of two successive models are compared according to the imposed criteria. In this study, two sets of the modal parameters obtained from two state space models of different orders are compared according to the following criteria.

$$
\begin{aligned}
\left|\frac{f^{(1)}-f^{(2)}}{f^{(1)}}\right| & <1 \% \\
\left|\frac{\xi^{(1)}-\xi^{(2)}}{\xi^{(1)}}\right| & <5 \% \\
1-\operatorname{MAC}\left(\phi^{(1)}, \boldsymbol{\phi}^{(2)}\right) & <2 \%
\end{aligned}
$$

where superscript $n$ is the model order; $f, \xi$ and $\phi$ are the frequency, the damping ratio and the mode shape vector, respectively. The modal assurance criterion (MAC) is used to quantify the similarity of two mode shapes vectors, and is defined by [2]

$$
\operatorname{MAC}\left(\phi^{(1)}, \phi^{(2)}\right)=\frac{\left|\phi^{(1)}{ }^{H} \phi^{(2)}\right|^{2}}{\left(\phi^{(1)^{H}} \phi^{(1)}\right)\left(\phi^{(2)^{H}} \phi^{(2)}\right)}
$$

A set of modal parameters is labeled as stable one if each inequality given by Equations (14) is satisfied. The idea is that the actual modal parameters show up consistently in models of different orders, whereas inconsistent spurious parameters do not. 


\section{INSTRUMENTATION SETUP AND GROUND MOTION RECORDS}

Seismic monitoring of structural systems is a component of the national earthquake hazard reduction program in the U.S. The seismic response of structures is obtained by recording vibrations during the events of strong ground motions. The recorded data are then analyzed by engineering and scientific communities to understand the actual behavior of the structures during earthquakes. Such data have been shown to be useful in assessing analysis and design procedures, and improving code provisions. These are main research objectives of the U.S. Geological Survey to instrument and monitor structures in the context of the earthquake hazard reduction program.

The California institute for telecommunications and information technology, Calit2, building site is one of more than 700 national strong motion monitoring stations in the U.S. The geographical coordinates of the Calit2 building location are $33.64^{\circ} \mathrm{N}$ latitude, $117.84^{\circ} \mathrm{W}$ longitude and $21 \mathrm{~m}$ altitude. In collaboration with the U.S. Geological Survey, the building and the surrounding soil were instrumented during construction with 43 force-balanced, uni-axial acceleration sensors. Thirty-one sensors were placed in the building at different levels, and 12 sensors were placed in the soil so that both structural response and the ground motion could be recorded. Of the 12 soil sensors, six were placed $3 \mathrm{~m}$ deep underground and six were placed in the free-field ground surface. Each floor was instrumented with at least five sensors to capture not only the transverse and longitudinal motions but also the torsional motions of the structure. Four sensors oriented in the vertical direction were mounted on the columns and shear walls at the ground level, and three more sensors oriented in the vertical direction were placed at the fourth-level ceiling to monitor the rocking motion of the structure. Figure 1 shows the sensor locations and their orientations. The data acquisition system, installed in the equipment room at the second floor of the building, consists of four recorders and a computer. A predetermined threshold level of the vibration amplitude was set for triggering the data acquisition system automatically. It can also be triggered manually. Since the building was constructed in 2004, four sets of ground motions and corresponding structural responses have been recorded, with a sampling rate of $200 \mathrm{~Hz}$, by automatic triggering due to four Southern California earthquakes. Further details of the ground motions are summarized in Table I, and Figures 2 and 3 show the time histories and the frequency contents of the ground motions, respectively.

The Calit2 building, located on the University of California, Irvine campus is a 4-story reinforced concrete structure with dimensions $26.82 \mathrm{~m} \times 76.20 \mathrm{~m}$ in plan, and $19.20 \mathrm{~m}$ height from the ground level. The building has four moment frames, spaced $8.84 \mathrm{~m}$ apart, in the longitudinal direction and eight moment frames, spaced $6.70 \mathrm{~m}$ apart, in the transverse direction. Lateral stiffness is provided by eight shear walls. Each floor has a reinforced concrete slab with a thickness of $0.20 \mathrm{~m}$. The overall weight and the fundamental period of the structure are estimated to be 12300 ton and $0.455 \mathrm{~s}$, respectively, in the design phase.

\section{IDENTIFICATION RESULTS}

The identification of the superstructure is of interest in this study. For the input, we consider the lateral sensor measurements (sensors 2, 3, 7 and 8) at the ground floor (rather than those at the down-hole or at the free-field) as the excitation. For the output, rigid body motions (the lateral translations and the rocking motions) were subtracted from the total response. In other words, the total response was decoupled into the structural and rigid body motions, and the structural motion was used for the system identification purpose. Using each data set separately, a corresponding state space model was identified for the building. Once a model is identified, it is assessed by comparing the predicted and measured responses. To this end, the model is used to predict the response to given input of the same data set. Two groups of the sensors, measuring the response in the different directions and at different levels, were chosen as references for response comparison. The first group of the sensors $(14,19$ and 26$)$, oriented in the transverse direction, is on the same vertical axis at different levels. The second group of the sensors $(18,25$, and 31), oriented in 


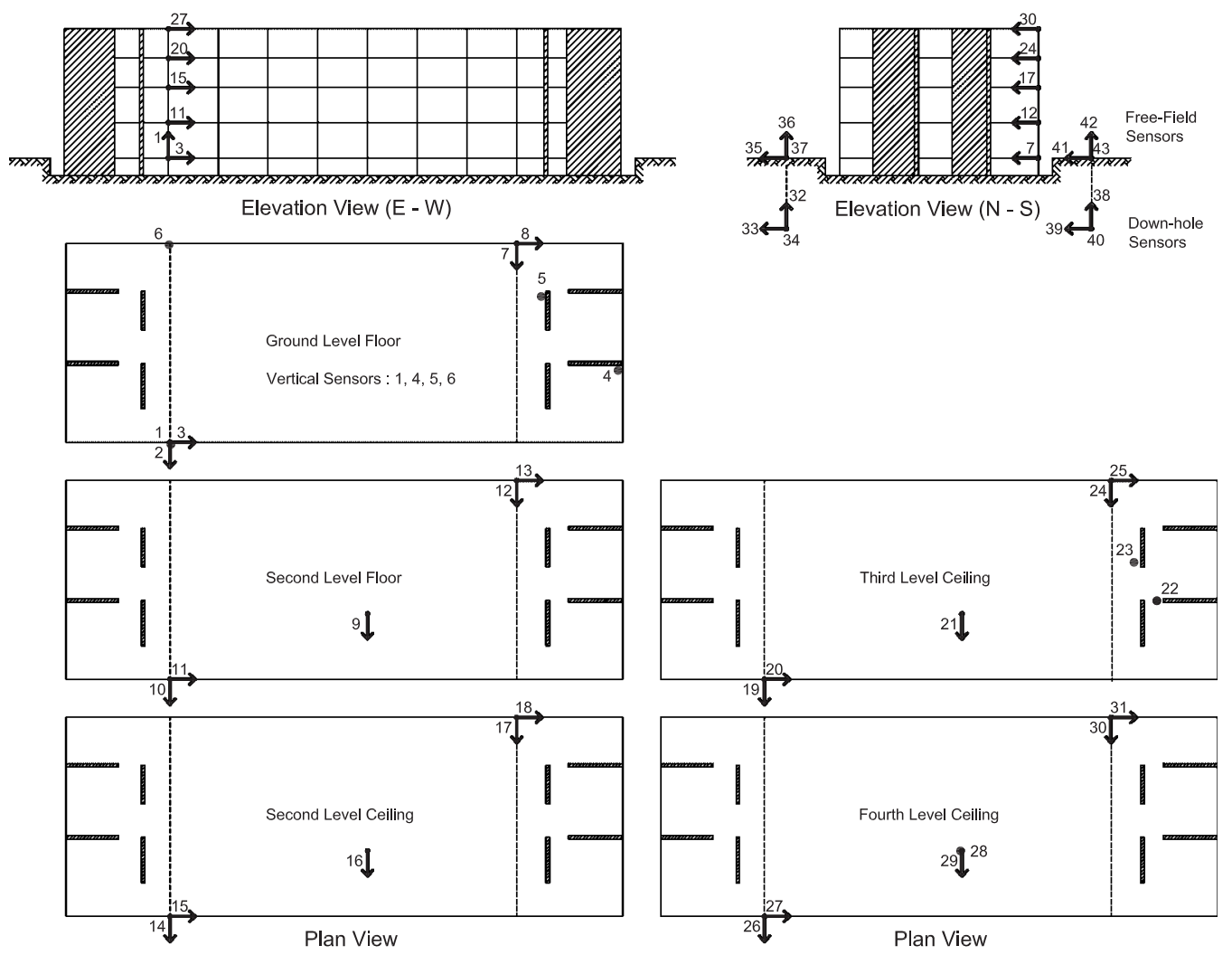

Figure 1. The Calit2 building sensor locations.

Table I. Recorded ground motions.

\begin{tabular}{lcccrr}
\hline & & & & \multicolumn{2}{c}{ PGA $\left(\mathrm{cm} / \mathrm{s}^{2}\right)$} \\
\cline { 5 - 7 } Earthquake & \multicolumn{1}{c}{ Date } & Magnitude & Distance to the epicenter $(\mathrm{km})$ & Longitudinal & Transverse \\
\hline Yucaipa & 16 June 2005 & 4.9 & 90 & 8.06 & 14.17 \\
San Clemente & 16 October 2005 & 4.9 & 135 & 2.45 & 4.47 \\
Chino Hills & 29 July 2008 & 5.4 & 35 & 54.85 & 47.85 \\
Inglewood & 17 May 2009 & 4.7 & 58 & 26.54 & 33.46 \\
\hline
\end{tabular}

the longitudinal direction, is on another vertical axis at different levels. The predicted responses, using a state space model of order six constructed from the Chino Hills data set, are compared with the measured responses for these two groups of sensors in Figures 4 and 5. The predicted responses show good agreement with the measured responses, particularly where the excitation level is strongest. The agreement between the measured and predicted responses for the state space models constructed from the other three earthquake data sets is similarly good. A state space model of order six thus represents well the underlying dynamics of this particular building. To quantify the error between the measured and predicted responses, a relative total error function, $J$, is defined by

$$
J=\left(\frac{\sum_{j} \sum_{k}\left(y_{j}(k)-\hat{y}_{j}(k)\right)^{2}}{\sum_{j} \sum_{k} y_{j}^{2}(k)}\right)^{1 / 2}
$$

For a particular data set, i.e. given the input-output data, the error function, $J$, can be regarded as an implicit function of the model order $n$. For different model orders, the normalized prediction errors were calculated and presented in Figure 6. As can be seen from Figure 6, J decreases monotonically, and after a certain model order, it does not change significantly as the model order 

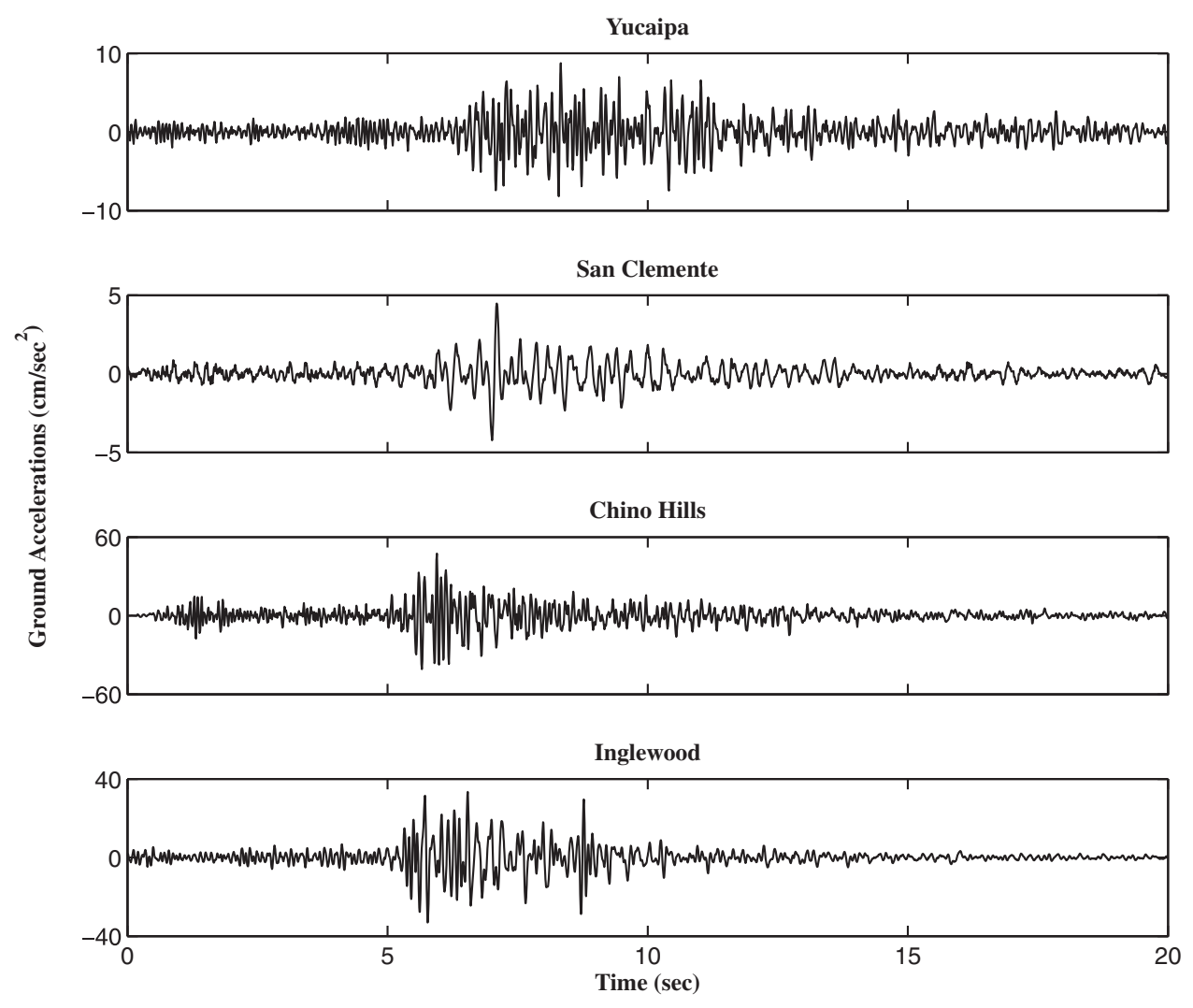

Figure 2. The time histories of the recorded ground motions.

increases. In other words, the measure of fit between the observed and predicted responses does not improve much after a certain model order, which implies that certain frequencies only are dominant in the response data. However, the determination of the model order based on the measure of fit is not a reliable approach especially when the contributions of some modes to the response are not significant.

The stabilization diagram alternatively is used to determine the model order and identify the modal parameters. Figure 7 shows the stabilization diagrams constructed for the Chino Hills data set. It is evident from the stabilization diagram that the well-excited mode parameters stabilize at low-order models, whereas poorly excited mode parameters stabilize at high model orders. The stabilization diagrams constructed for all four data sets show that the first three modes are well excited, thus dominant in the data. The identified first mode is the bending in the longitudinal direction, whereas the second and third ones are the coupled bending modes in the transverse and longitudinal directions. The frequencies and damping ratios identified are summarized in Table II.

\subsection{Discussion of results}

The frequencies and damping ratios identified, using the state space models constructed for each of the earthquake input-output data sets, vary depending on the earthquake data set considered. The first natural frequency varies between $2.50 \mathrm{~Hz}$ in the Chino Hills event and $2.87 \mathrm{~Hz}$ in the San Clemente event, whereas the associated damping ratios vary between 4.92 and $2.45 \%$ of critical damping for the same data sets. The variation in the modal parameters implicitly indicates nonlinear behavior of the structure within the range of earthquake excitation considered in this 

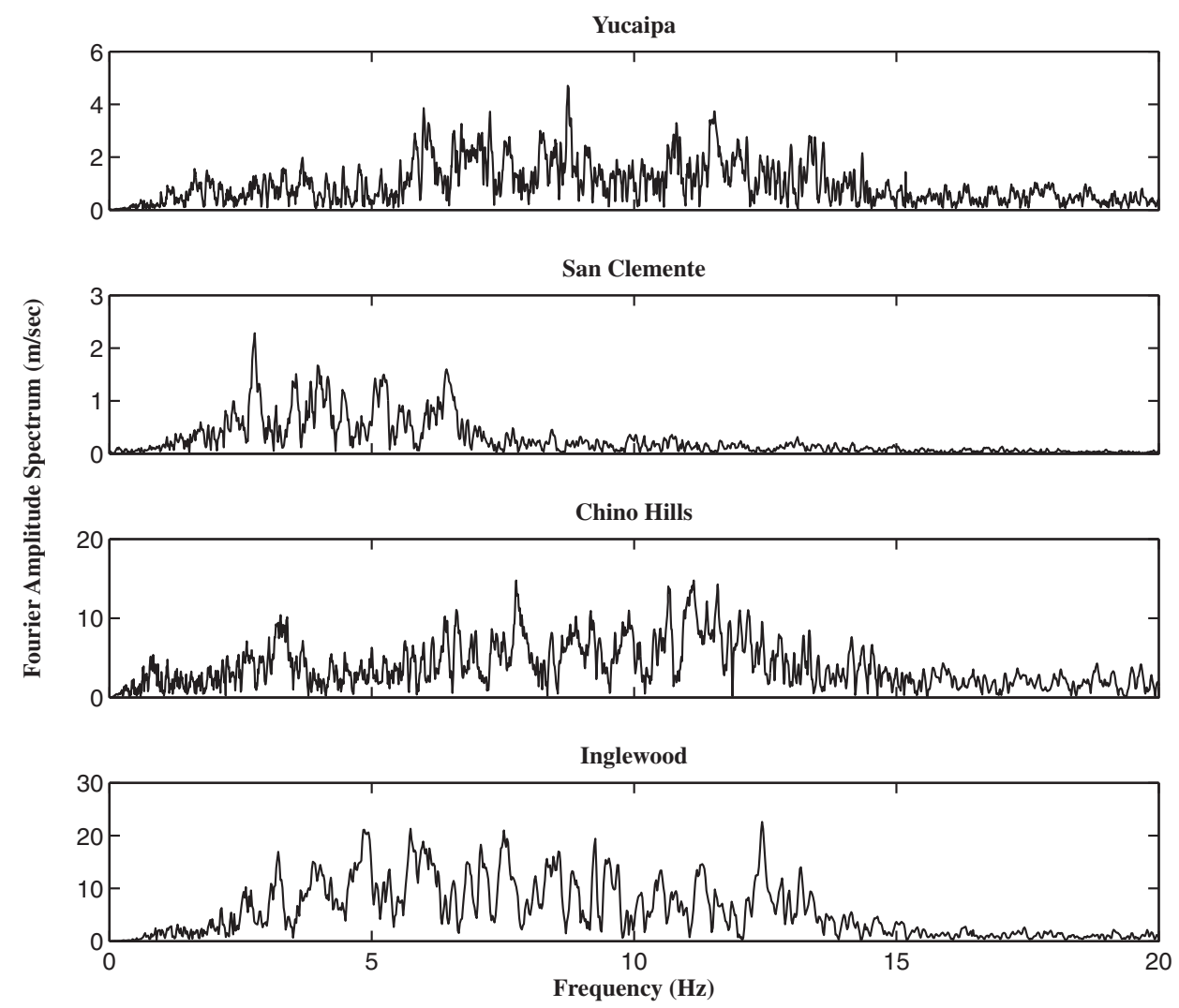

Figure 3. The frequency contents of the recorded ground motions.

study. However, the individual linear state space models for each data set represent the building dynamics for each level of excitation well.

The response frequencies and damping values are plotted against the peak ground accelerations in Figure 8. As the amplitude of ground motion increases, joints and structural interfaces are mobilized more completely thereby reducing the response frequency and increasing the level of damping - the building becomes apparently less stiff while exhibiting a greater capacity to dissipate energy. This is consistent with the findings in [22] when studying the dynamic response of the Di-Wang Tower in China.

Given the amplitude-dependent response, it is unreasonable to expect any one of the four state space models to accurately predict the response due to other different excitation levels. However where the level of excitation due to different earthquakes is similar, the models perform very satisfactorily - in this study, the levels of excitation for the Chino Hills and the Inglewood earthquakes are similar. Figure 9 compares the predicted response due to the Chino Hills earthquake using the state space model identified by the Inglewood data set to the measured response of the Chino Hills data set. It is evident from Figure 9 that the agreement between the predicted and measured responses is very good.

It is also evident, from Figure 8, that within the range of earthquake excitation considered, $0.005 \mathrm{~g}$ to $0.074 \mathrm{~g}$, there is a practically linear relationship between the amplitude of ground acceleration and the frequency of response. The minimum correlation coefficient, $R^{2}$, for linear regressive lines fitted to the frequency data is 0.93 . The relationship between amplitude of ground acceleration and damping is less strong $\left(R_{\min }^{2}=0.55\right)$.

A practical application of the state space models developed is the ability to use the extracted modal parameters-frequencies, damping values and mode shapes-as indicators of consistent structural response in future earthquakes. Within the range of $0.005 \mathrm{~g}$ to $0.074 \mathrm{~g}$ the expectation is 

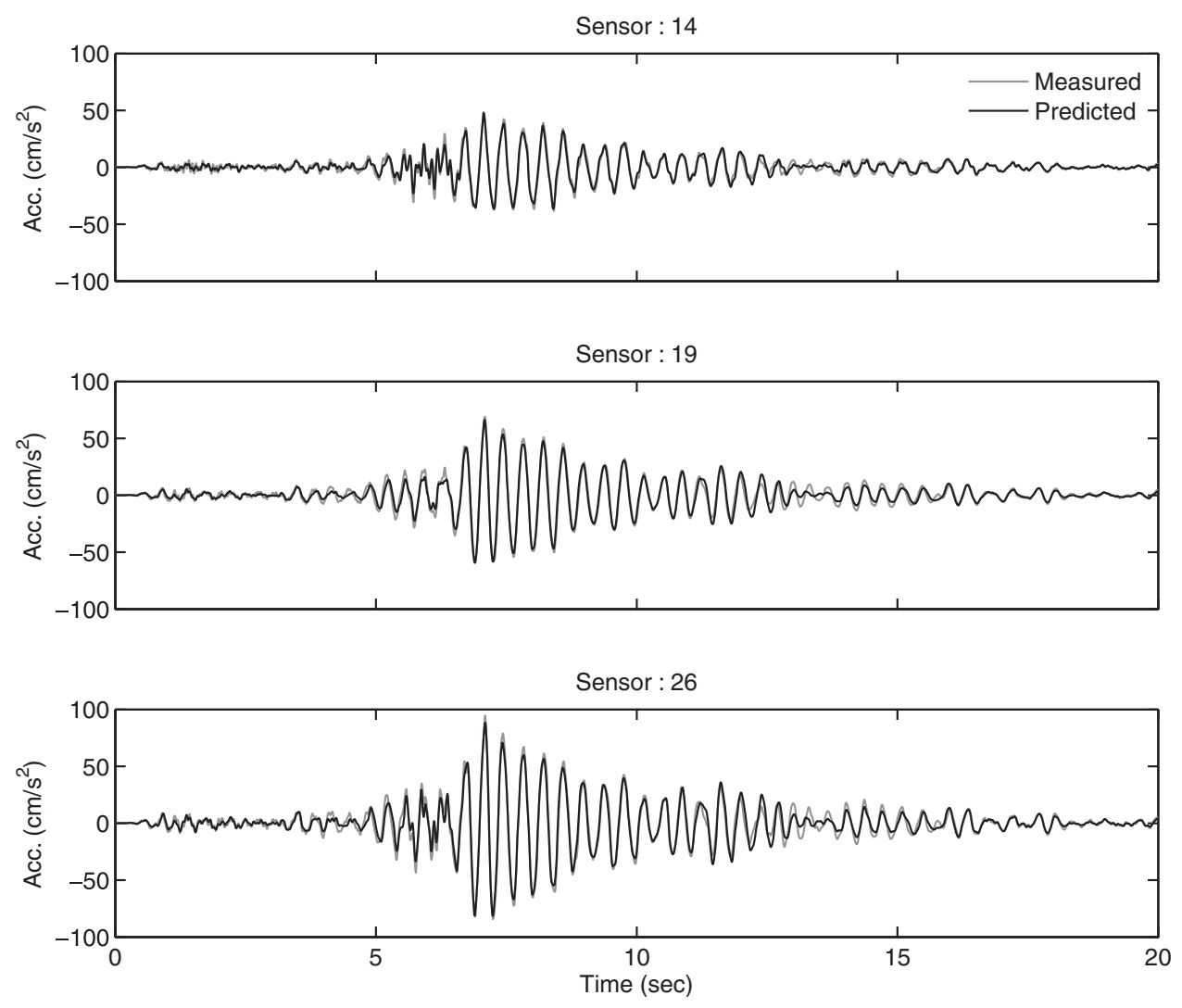

Figure 4. Comparison of the measured and predicted responses: The Chino Hills data set.

that the structure's response, determined from a new state space model, should be consistent with the linear relationships plotted in Figure 8. Further earthquake events will clearly add confidence to the current data sets and also potentially enable the relationship to be extended to a wider range of ground excitations.

Equally, within this range, a rapid assessment of the consistency of structural response is possible by quantification of the magnitude of ground acceleration, from the ground sensors, and extraction of the modal frequencies and damping values from the structural response and plotting this data against the model data already reported in Figure 8. In the case of this particular structure, if the Iglewood data were omitted and linear regression analysis is used with the three other earthquake data sets, the expected frequencies of response for the first three modes are found to be within $2.5 \%$ of those experienced during that earthquake-in other words having only prior knowledge of the response to the three other earthquakes, the frequency response to the Inglewood earthquake could be deemed to be consistent with the expected structural response. While the relationships between frequency, damping and ground excitation level are specific to this particular test structure, similar relationships could be developed for other structures using the state space modeling- the requirement being that both excitation and response measurement data are available.

\section{CONCLUSIONS}

In this paper, four state space models of a 4-story building have been identified from multiple sets of earthquake response data. Each model accurately replicates the building dynamic response for the earthquake from which it was constituted. Once constituted these mathematical models were successfully used to extract the dominant frequencies, modes of vibration and associated damping values for the building due to the four earthquake data sets. 

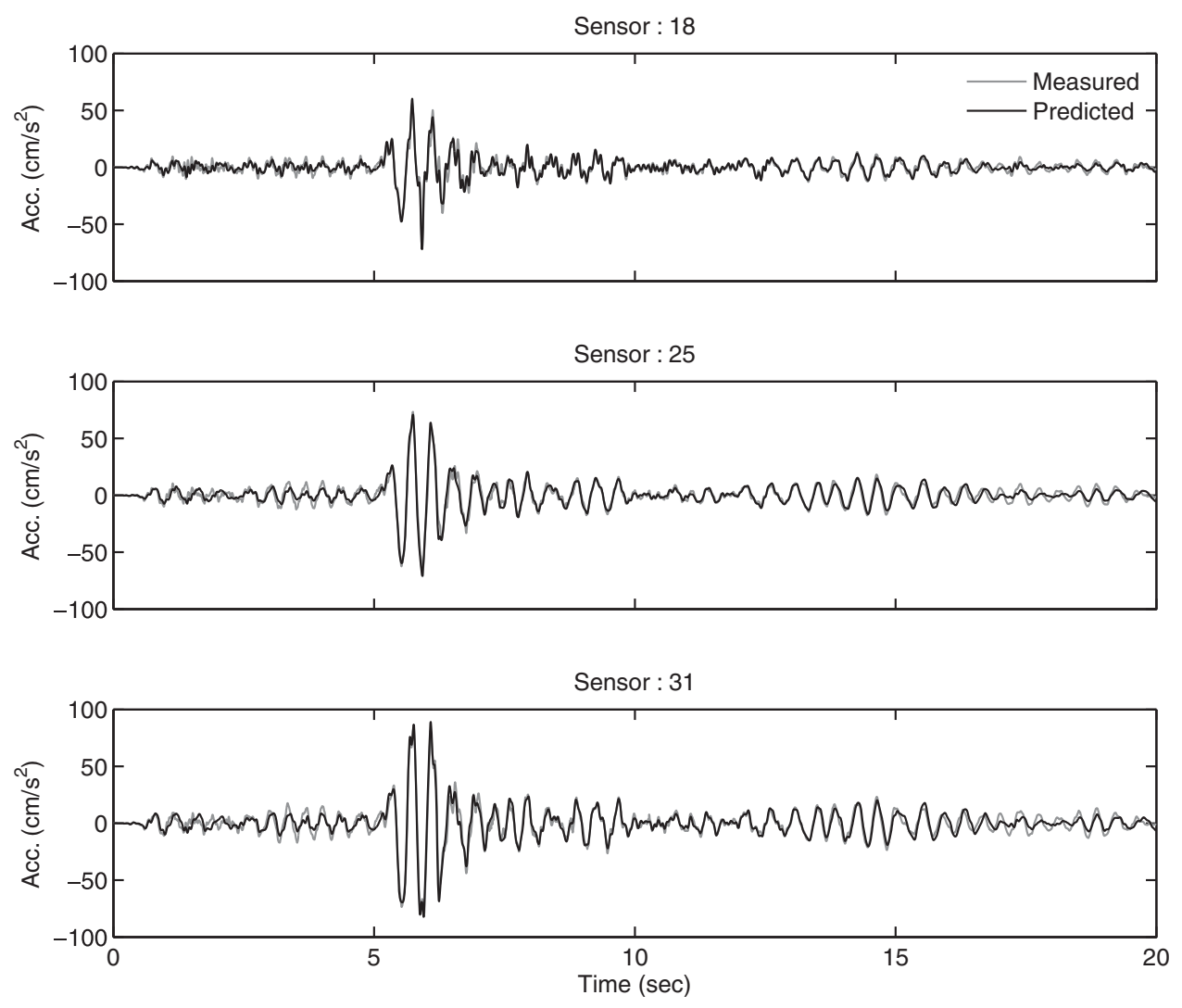

Figure 5. Comparison of the measured and predicted responses: The Chino Hills data set.
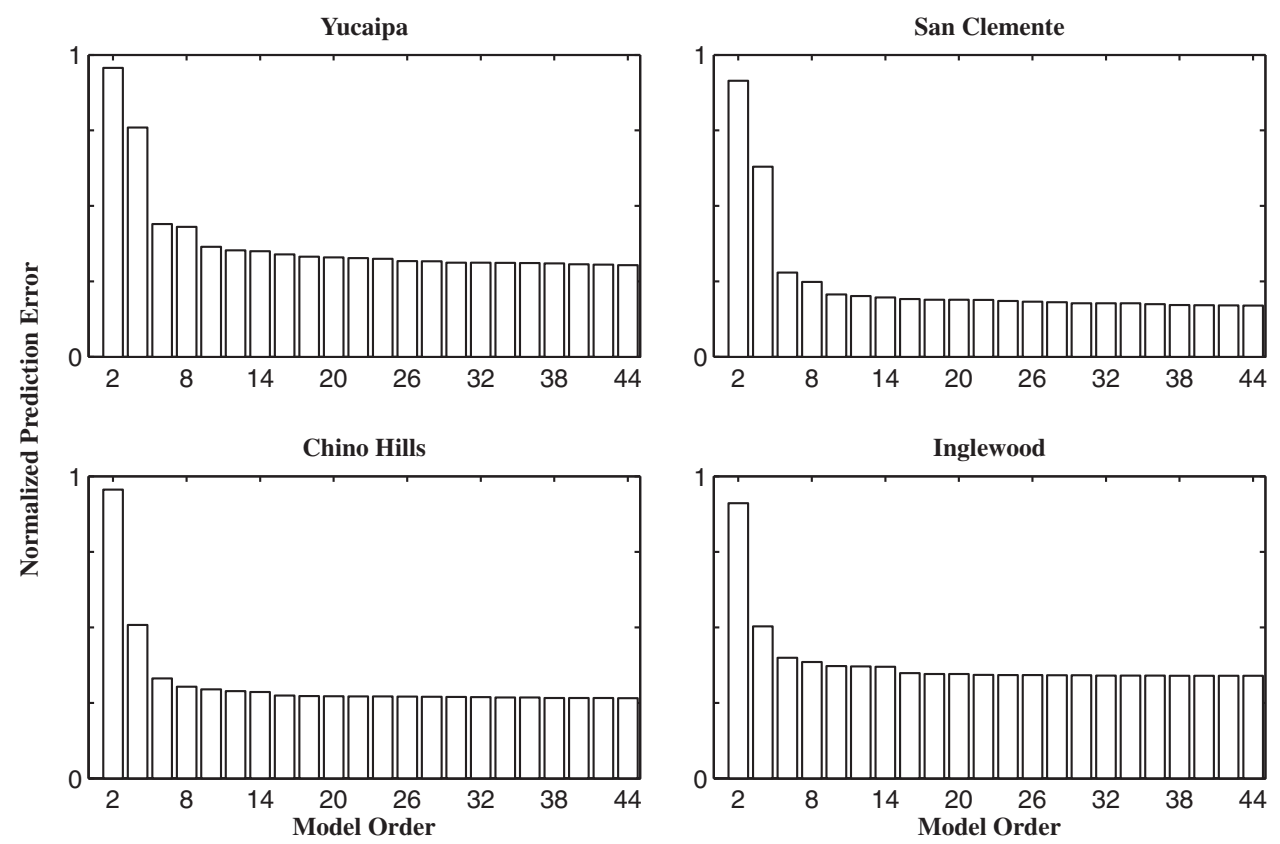

Figure 6. Normalized prediction error as a function of model order. 


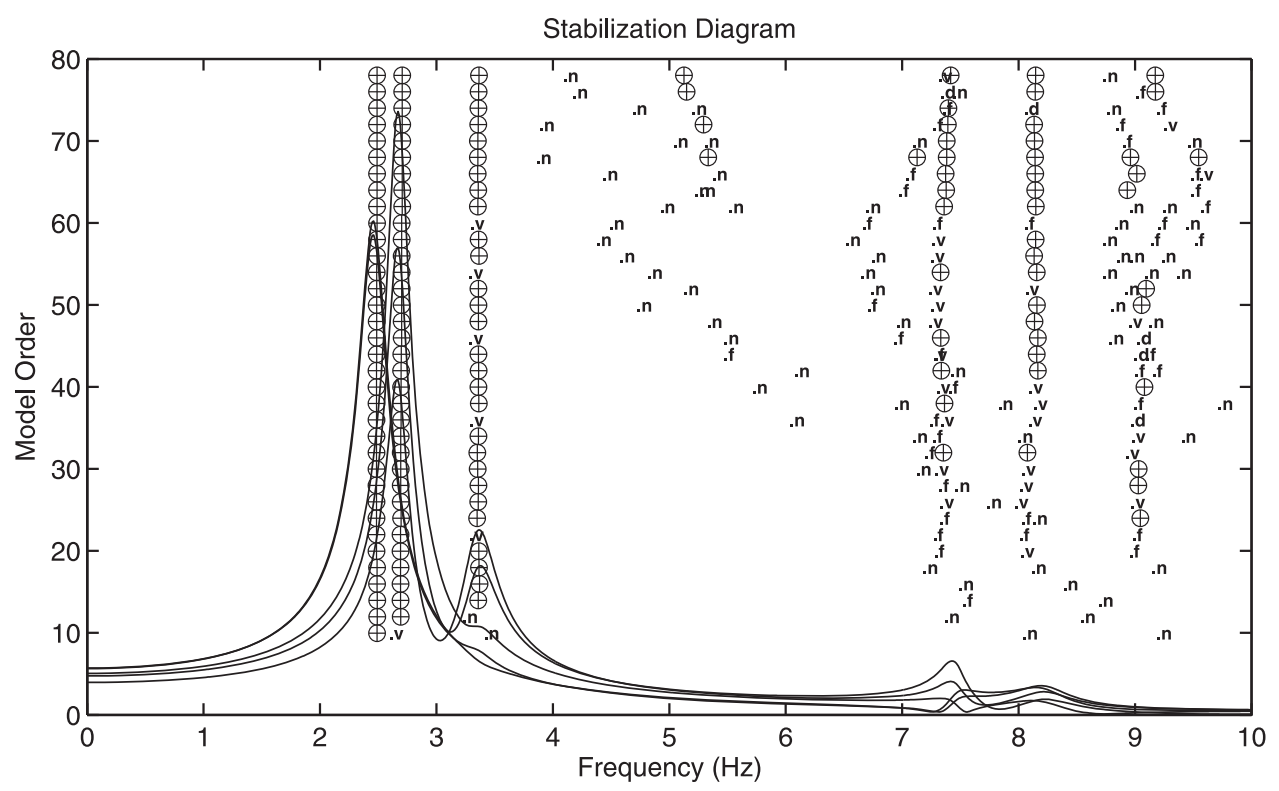

Figure 7. The stabilization diagram for the Chino Hills data set. The labels are: ' $\oplus$ ' for a stable pole; '.v' for a pole with stable frequency and vector; '.d' for a pole with stable frequency and damping; '.f' for pole with stable frequency; and '.n' for a new pole.

Table II. Identified modal frequencies $f$ and damping ratios $\xi$

\begin{tabular}{|c|c|c|c|c|c|c|c|c|}
\hline \multirow[b]{2}{*}{ Modes } & \multicolumn{2}{|c|}{2005 Yucaipa } & \multicolumn{2}{|c|}{2005 San Clemente } & \multicolumn{2}{|c|}{2008 Chino Hills } & \multicolumn{2}{|c|}{2009 Inglewood } \\
\hline & $f(\mathrm{~Hz})$ & $\xi(\%)$ & $f(\mathrm{~Hz})$ & $\xi(\%)$ & $f(\mathrm{~Hz})$ & $\xi(\%)$ & $f(\mathrm{~Hz})$ & $\xi(\%)$ \\
\hline 1 & 2.77 & 4.36 & 2.87 & 2.45 & 2.50 & 4.92 & 2.59 & 4.50 \\
\hline 2 & 2.92 & 3.48 & 2.94 & 2.82 & 2.68 & 4.10 & 2.74 & 3.84 \\
\hline 3 & 3.55 & 3.50 & 3.59 & 1.65 & 3.43 & 3.85 & 3.46 & 3.75 \\
\hline
\end{tabular}

Linear state space models of higher order were found to give a better correlation between the measured and predicted responses. However, it was also shown that beyond a certain model threshold the measure of fit does not improve significantly-for these particular data sets, models with order of six were found to be sufficient. While the selection of model order, based on the magnitude of an error function defined between the measured and predicted responses alone, is somewhat subjective it was found that the model order selection can also be instructively informed by construction and analysis of the stabilization diagrams for the various data sets.

The state space models derived were different for earthquakes of different magnitudes. As a result, the dynamic characteristics of the building were found to be different during earthquakes of different magnitudes. Larger amplitude earthquakes resulted in reduced natural frequencies and increased damping. This nonlinear building response explains why any one of the four state space models does not precisely capture the response to another one of the earthquakes.

However, taken cumulatively the four individual models give an insight into the extent and type of amplitude dependent response. Over the range of ground motion excitation considered, $0.005 \mathrm{~g}$ to $0.074 \mathrm{~g}$, it was found that the first three natural frequencies strongly correlate with the peak ground acceleration for this building. The associated damping values also exhibited amplitude dependency in this range, but the correlation was not as strong. The practical use of this finding is that a rapid assessment of the consistency of building response due to an earthquake can be made. In the case of the Inglewood earthquake the projected frequencies of response, over the first three modes, were found to be within $2.5 \%$ of those measured when the amplitude-dependent 

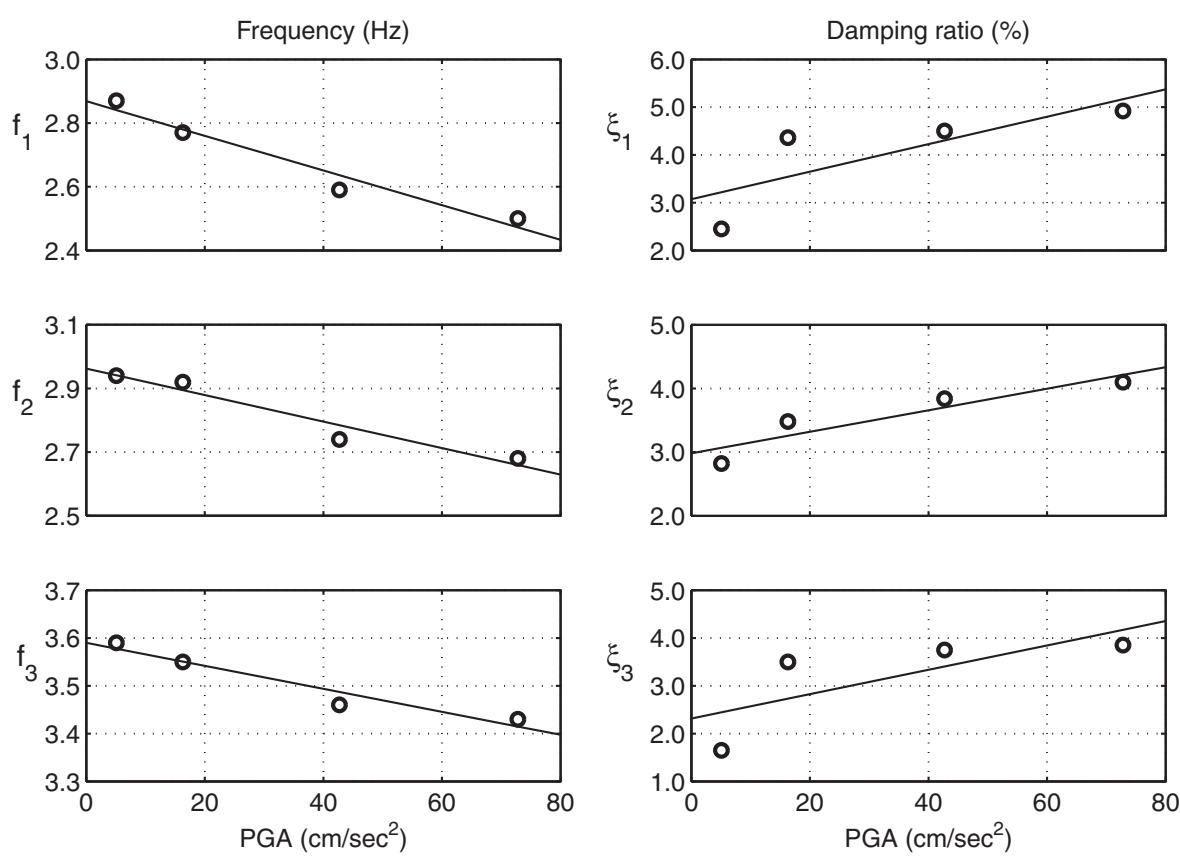

Figure 8 . The variation of the modal parameters with the peak ground accelerations.
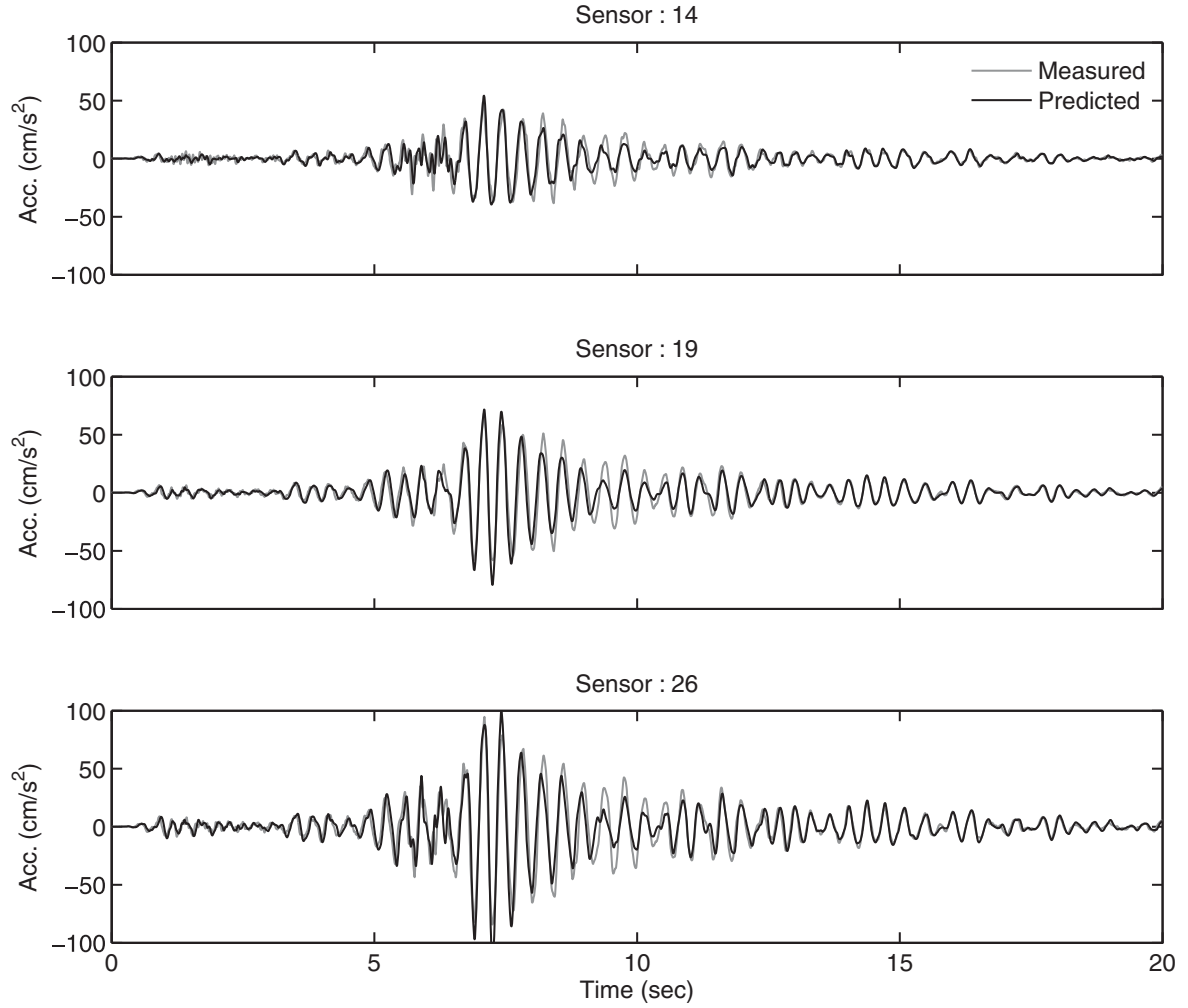

Figure 9. Comparison of the measured and predicted responses: The Chino Hills and Inglewood data sets.

response is defined by the other three earthquake data sets alone. Future data sets can thus be used for performance evaluation and/or to extend or confirm this correlation between amplitude of excitation and dynamic response. 


\section{ACKNOWLEDGEMENTS}

The authors would like to acknowledge Calit2 of UC Irvine and the U.S. Geological Survey for providing funding to instrument the Calit2 building. The authors also thank structural engineer Mick Wilson for discussions and providing the basic design parameters of the building.

\section{REFERENCES}

1. Ljung L. System Identification: Theory for the Users (2nd edn). Prentice-Hall: Upper Saddle River, NJ, 1999.

2. Ewins DJ. Modal Testing: Theory, Practice and Application. Research Studies Press: Baldock, Hertfordshire, U.K., 2000.

3. James GH, Carne TG, Lauffer JP. The natural excitation technique (NExT) for modal parameter extraction from operating wind turbines. Report No. SAND92-1666, UC-261, Sandia National Laboratories, Sandia, NM, 1993.

4. Brincker R, Zhang LM, Andersen P. Modal identification of output-only systems using frequency domain decomposition. Smart Materials and Structures 2001; 10(3):441-445.

5. Peeters B, De Roeck G. Reference-based stochastic subspace identification for output-only modal analysis. Mechanical Systems and Signal Processing 1999; 13(6):855-878.

6. Peeters B, De Roeck G. Stochastic system identification for operational modal analysis: a review. Journal of Dynamic Systems, Measurement and Control-Transactions of the ASME 2001; 123(4):659-667.

7. Udwadia FE, Trifunac MD. Time and amplitude dependent response of structures. Earthquake Engineering and Structural Dynamics 1974; 2:359-378.

8. Trifunac MD, Ivanovic SS, Todorovska, MI. Apparent periods of a building. I: Fourier analysis. Journal of Engineering Mechanics (ASCE) 2001; 127(5):517-526.

9. Trifunac MD, Ivanovic SS, Todorovska, MI. Apparent periods of a building. II: time-frequency analysis. Journal of Engineering Mechanics (ASCE) 2001; 127(5):527-537.

10. Todorovska MI, Trifunac MD. Impulse response analysis of the Van Nuys 7-story hotel during 11 earthquakes and earthquake damage detection. Structural Control and Health Monitoring 2008; 15(1):90-116.

11. Hong AL, Betti R, Lin CC. Identification of dynamic models of a building structure using multiple earthquake records. Structural Control and Health Monitoring 2009; 16(2):178-199.

12. Beck JL, Jennings PC. Structural identifcation using linear models and earthquake records. Earthquake Engineering and Structural Dynamics 1980; 8(2):145-160.

13. Safak E. Adaptive modeling, identification, and control of dynamic structural systems. II: applications. Journal of Engineering Mechanics (ASCE) 1989; 115(11):2406-2426.

14. Loh CH, Lin HM. Application of off-line and on-line identification techniques to building seismic response data. Earthquake Engineering and Structural Dynamics 1996; 25(3):269-290.

15. Smyth AW, Pei J-S, Masri SF. System identification of the Vincent Thomas suspension bridge using earthquake records. Earthquake Engineering and Structural Dynamics 2003; 32(3):339-367.

16. Juang J-N, Pappa RS. An eigensystem realization algorithm for model parameter identification and model reduction. Journal of Guidance, Control and Dynamics 1985; 8(5):620-627.

17. Juang J-N, Phan M, Horta LG, Longman RW. Identification of Observer/Kalman filter Markov parameters: theory and experiments. Journal of Guidance, Control and Dynamics 1993; 16(2):320-329.

18. Ho BL, Kalman RE. Effective construction of linear state variable models from input/output data. Regelungstechnik 1966; 14:545-548.

19. Lus H, Betti R, Longman RW. Identification of linear structural systems using earthquake-induced vibration data. Earthquake Engineering and Structural Dynamics 1999; 28(11):1449-1467.

20. Lus H, Betti R, Longman RW. Obtaining first-order predictive models of linear structural systems. Earthquake Engineering and Structural Dynamics 2002; 31(7):1413-1420.

21. Juang J-N. Applied System Identification. Prentice-Hall: Englewood Cliffs, NJ, 1994.

22. Li QS, Yang K, Zhang N, Wong CK, Jeary AP. Field measurements of amplitude-dependent damping in a 79-story tall building and its efects on the structural dynamic responses. Structural Design of Tall Buildings 2002; 11(2):129-153. 